\title{
Subacute Sclerosing Panencephalitis (SSPE): A Rare Case Report
}

\author{
MOHAMMAD RAFIQUL ISLAM, ${ }^{1}$ MAMUNUR RASHID BHUIYAN, ${ }^{2}$ SUDI P RANJ AN DEB, ${ }^{1}$ AHMEDUL KABI R ${ }^{3}$
}

\begin{abstract}
:
Subacutesclerosingpanencephalitis(SSPE) is a late complication of measles virus infection. It presents with psychiatric manifestation, progressive dementia, myoclonic jerk and other focal neurological signs. The diagnosis is based upon characteristics clinical manifestation, the presence of characteristic periodic EEG discharge, and demonstration of raised antibody titer against measles in plasma and cerebrospinal fluid. In our case patient presented with behavioral change, myoclonic jerk, characteristic EEG and elevated level of antibody both in serum and CSF.
\end{abstract}

Key words: Subacutesclerosingpanencephalitis; myoclonic jerk; electroencephalography.

\section{Introduction:}

Subacutesclerosingpanencephalitis (SSPE) was first described by Dawson in 1933-1934 as sub acute inclusion encephalitis. It is a rare progressive, invariably fatal longterm complication of measles infection. The latency period between acute measles and first symptoms of SSPE is usually 4 to 10 years but ranges from 1 month to 27 years. ${ }^{1}$ Although SSPE affects mostly children younger than 12 years, interestingly, there is a considerable increase in the number of adult cases of SSPE. ${ }^{2,3}$

\section{Case report:}

A 15 Year old boy of $9^{\text {th }}$ grade, hailing from Jamalpur, admitted into DMCH (Dhaka Medical College Hospital) with the complaints of altered behavior for last 7 months and jerky movement of the whole body for same duration. Seven months back, before admission, he developed fever which was high grade continued in nature, with chills and rigor, subsided with anti pyretic. Fever was associated with maculopapular rash all over the body which disappeared after few days and was also associated with cough, coryza and conjunctivitis. Fever subsided after 5 days; thereafter he developed severe vomiting not responding to medication. Patient's mother gave history about infrequent small jerky movement of the whole body from the onset of fever but this abnormal movement gradually increased in frequency and severity. Along with abnormal movement, patient also developed abnormal behavior in the form of repetition of

1. Assistant Professor, Department of Medicine, Dhaka Medical College\& Hospital

2. Medical Officer, Department of Medicine, Dhaka Medical College \& Hospital

3. Associate Professor, Department of Medicine, Dhaka Medical College\& Hospital

Correspondence : Mohammad Rafiqul Islam. Assistant Professor, Department of Medicine, Room-503, Dhaka Medical College \& Hospital-2, Dhaka. Email: drrafiq73@yahoo.com. Mob: 01753199796. same word, forgetfulness, smiling without any reason and poor school performance. Patient had no history of unconsciousness, convulsion, hemiparesis, visual disturbance or bladder bowel incontinence. Three months later, abnormal jerky movements became severe and more frequent, predominantly on the left side but never during sleep. Patient also developed generalized rigidity. He came from a low socioeconomic condition and has incomplete vaccination status. His parents could not specify whether measles vaccine was given or not. On query, his mother gave history of maculopapular rash associated with fever at the age of 5 yrs. On general examination patient was bed ridden due to generalized rigidity and frequent jerky movements. Patient was smiling on query. On nervous system examination higher psychic function couldn't be evaluated. Pupils were normal in size and were reacting to light. Signs of meningism were absent. Deep tendon reflex was present but diminished, bilateral plantar response was flexor. Frequent repetitive jerky movements of the whole body more on the left side were present. . All other systemic examination revealed normal. Slit lamp examination of eyes revealed no KF ring. MRI of brain was normal. EEG showed repeated bouts of high voltage sharp and slow waves seen in both the hemispheres more on provocation suggestive of generalized epileptiform discharge. Measles virus antibody in blood IgG positive, IgM negative. Urinary copper $<20.0 \mathrm{mcg} / \mathrm{l}$ ( $>100 \mathrm{mcg} / \mathrm{l}$ in Wilson's disease), Serum copper $89 \mathrm{mcg} / \mathrm{l}(70-140 \mathrm{mcg} / \mathrm{l})$, concentration of ceruloplasmin $22 \mathrm{mg} / \mathrm{dl}$ (15-60 mg/dl). CSF showed protein 30 g/dl, Sugar - $64.8 \mathrm{mg} / \mathrm{dl}$, WBC- total count- 02 /cmm. Lymphocyte $-100 \%$.Gram stain and AFB stain were negative. CSF for ADA: $12.9 \mathrm{u} / \mathrm{l}$. Measles virus Antibody in CSF IgG: Positive (AI: 4.26), Ref value (AI: 1.0). IgM: Negative (AI: 0.2 ), Ref value (AI: 1.0 ).

\section{Discussion:}

In the classical form of SSPE, slow progression of neurologic symptoms goes through four characteristic stages: (I) slowly 
evolving behavioral and intellectual deterioration; (II) various types of involuntary movements; (III) severe pyramidal and extrapyramidal hypertonus and disappearance of hyperkinesias; and (IV) chronic vegetative state and death. Stage I may last from several weeks to several years, stage II typically lasts from three months to one year. Stages III and IV often last from six months to one year. ${ }^{4,}{ }^{5}$ In our case, patient's vaccination was incomplete and had a fever with maculopapular rash at the age of 5 years. No work up was done at that time. This time patient presented with high grade continued fever, maculopapular rash, cough, coryza and conjunctivitis and thereafter developed movement and behavioral disorder. Maculopapular rash are frequently seen in viral and immunological illness. Viral causes are rubella, rubeola, roseola and erythema infectiosum. Patient also developed abnormal behavior in the form of repetition of same word, forgetfulness, smiling without any reason and poor school performance. In SSPE abnormal behavior develops in the first stage. Many a times it regarded as psychiatric illness. This was happened to our patient. Patient also presented with small jerky movement of the whole body infrequently but this abnormal movement gradually increased in frequency and severity. Three months later, abnormal jerky movements became severe and occurred more frequently, predominantly on the left side. Generalized rigidity was also present. Diagnosis of SSPE is based on a positive history of measles infection, clinical picture, typical electroencephalographic finding (suppression burst pattern), elevated serum and CSF anti measles antibody titer. ${ }^{6,7}$ In our case patient had incomplete vaccination history, history of fever suggestive of measles at the age of five years, behavioral and neurological manifestation, characteristic EEG with rise if anti measles Ig $\mathrm{G}$ in both serum and CSF (cerebro spinal fluid) were present. There was a confusion that patient had similar type of fever at the age of 5 years and 15 years. It was difficult to ascertain which one was real measles. As SSPE is a late complication of measles, we thought fever at the age of 5 years was measles. Reverse transcriptase polymerase chain reaction technique and brain biopsy might be useful in confirming the diagnosis in SSPE with negative CSF findings. ${ }^{8} \mathrm{MRI}$ is more sensitive in detecting white matter abnormalities. In the early stages of the disease, cerebral MRI shows lesions usually involving parieto-occipital cortico-subcortical regions asymmetrically. In time, symmetric periventricular white matter changes become more prominent. However, more recently Aydin et al. ${ }^{9}$ described that MRI findings could be normal with SSPE.Treatment of SSPE is still on research. Antiviral agents, such as amantadine and ribavirin or imunomodulators such as isoprinosine, interferon, and immunoglobulin have been used. But in our patient due to economical constrain we gave him phenytoin and clonazepam to control jerky movements but unfortunately patient responded a little.

\section{Conclusion:}

When a young patient present with neuropsychiatric manifestation,focal neurological deficit; besides Wilsons disease and post encephalitic syndrome, Subacute sclerosingpanencephalitis should be kept as a differential diagnosis. Although SSPEis a very rare disease, still on the background of characteristic fever, neuropsychiatric manifestation and classical EEG findings, diagnosis is easy.

\section{Conflict of interest: None.}

\section{References:}

1. H, Andrews N, Brown KE, Miller E. Review of the effect of measles vaccination on the epidemiology of SSPE. Int $\mathrm{J}$ Epidemiol 2007; 36: 1334-1348.

2. Frings M, Blaeser I, Kastrup. Adult-onset subacute sclerosingpanencephalitis presenting as a degenerative dementia syndrome. J Neurol 2002; 249(7): 942-3.

3. Gokcil Z, Odabasi Z, Demirkaya S, Eroglu E, Vural O. AlphaInterferon and Isoprinosine in adult-onset subacute sclerosingpanencephalitis. J Neurol Sci 1999; 162(1): 62-4.

4. Adams RD, Victor M. Subacutesclerosingpanencephalitis. In: Lamsback WY, Navrozov M (eds). Principles of Neurology (5th ed). New York: McGraw-Hill; 1993: 656657.

5. Dyken PR. Viral diseases of the nervous system. In: Swaiman KF (ed). Pediatric Neurology, Vol 1. St Louis: CV Mosby; 1989: 499-501.

6. Dyken PR. Subacute sclerosingpanencephalitis Currentstatus. Neurol Clin 1985; 3: 179-196.

7. Griffin DE. Viral infections and related disorders. In: Scheld WM, Whithley RJ, Durack DT (eds). Infections of the Central Nervous System. Philadelphia, New York: Lippincott-Raven; 1997: 47-57.

8. Chung BH, Wong VC, et al. Acute fulminant Subacute sclerosingpanencephalitis with absent measles and PCR studies in cerebrospinal fluid. Pediatr Neurol 2004; 31: 222-224.

9. Aydin K,Okur O,Tatli B,Sarwar SG,Ozturk C \& Dilber C. Reduced Gray Matter Volume in the Frontotemporal Cortex of Patients with Early Subacute Sclerosing Panencephalitis. Am J Neuroradiol 2009; 30( 2): 271-275. 